\title{
Similarities and differences between the immunopathogenesis of COVID-19-related pediatric multisystem inflammatory syndrome and Kawasaki disease
} Claudia Fortuny, ${ }^{3,4,8,9,10}$ María Rios-Barnes, ${ }^{8}$ Joan Sanchez-de-Toledo, ${ }^{3,11,12}$ Mónica Girona-Alarcón, ${ }^{13}$ Juan Manuel Mosquera, ${ }^{3,5}$ Silvia Ricart, ${ }^{4,6}$ Cristian Launes, ${ }^{3,4,6,9}$ Mariona Fernández de Sevilla, ${ }^{6,9}$ Cristina Jou, , 34,15 Carmen Muñoz-Almagro, ${ }^{3,9,16,17}$ Eva González-Roca, ${ }^{18,19}$ Andrea Vergara, ${ }^{18,20}$ Jorge Carrillo, ${ }^{21}$ Manel Juan, ${ }^{2,4,19}$ Daniel Cuadras, ${ }^{3,22}$ Antoni Noguera-Julian, ${ }^{3,4,8,9,10}$ Iolanda Jordan, ${ }^{3,4,9,13}$ and Laia Alsina ${ }^{1,2,3,4}$

'Allergy and Clinical Immunology Department, Hospital Sant Joan de Déu (HSJD), Barcelona, Spain. ${ }^{2}$ Clinical Immunology Unit, HSJD-Hospital Clinic de Barcelona (HCB), Barcelona, Spain. ${ }^{3}$ Institut de Recerca Sant Joan de Déu, Barcelona, Spain. ${ }^{4}$ Universitat de Barcelona, Barcelona, Spain. ${ }^{5}$ Pediatric Rheumatology Department and ${ }^{6}$ Pediatrics Department, HSJD, Barcelona, Spain. ${ }^{7}$ Pediatric Rheumatology Department, Hospital Parc Tauli, Sabadell, Spain. ${ }^{8}$ Infectious Diseases Department, HSJD, Barcelona, Spain. ${ }^{9}$ CIBER of Epidemiology and Public Health, Madrid, Spain. ${ }^{10}$ Translational Research Network in Paediatric Infectious Diseases (RITIP), Madrid, Spain. "Cardiology Department, HSJD, Barcelona, Spain. ${ }^{2}$ Department of Critical Care Medicine, University of Pittsburgh, Pittsburgh, Pennsylvania, USA. ${ }^{13}$ Pediatric Intensive Care Unit, and ${ }^{14}$ Pathology Department and Biobank, HSJD, Barcelona, Spain. ${ }^{15}$ Center for the Biomedical Research on Rare Diseases (CIBERER), ISCIII, Madrid, Spain. ${ }^{15}$ Department of Medicine, Universitat Internacional de Catalunya, Barcelona, Spain. ${ }^{17}$ Molecular Microbiology Department, HSJD, Barcelona, Spain. ${ }^{18}$ Molecular Biology Core, and ${ }^{19}$ Department of Immunology, El Centro de Diagnóstico Biomédico (CDB), HCB-IDIBAPS, Barcelona, Spain. ${ }^{20}$ Microbiology Department, CDB, HCB, Barcelona, Spain. ${ }^{21}$ IrsiCaixa AIDS Research Institute, Hospital Cermans Trias i Pujol, Badalona, Catalonia, Spain. ${ }^{22}$ Statistical Department, Fundació Sant Joan de Déu, Barcelona, Spain.

\begin{abstract}
Multisystem inflammatory syndrome associated with the SARS-CoV-2 pandemic has recently been described in children (MIS-C), partially overlapping with Kawasaki disease (KD). We hypothesized that (a) MIS-C and prepandemic KD cytokine profiles may be unique and justify the clinical differences observed, and (b) SARS-CoV-2-specific immune complexes (ICs) may explain the immunopathology of MIS-C. Seventy-four children were included: 14 with MIS-C, 9 patients positive for SARS-CoV-2 by PCR without MIS-C (COVID), 14 with prepandemic KD, and 37 healthy controls (HCs). Thirty-four circulating cytokines were quantified in pretreatment serum or plasma samples and the presence of circulating SARS-CoV-2 ICs was evaluated in MIS-C patients. Compared with HCs, the MIS-C and KD groups showed most cytokines to be significantly elevated, with IFN- $\gamma$-induced response markers (including IFN- $\gamma$, IL-18, and IP-10) and inflammatory monocyte activation markers (including MCP-1, IL-1 $\alpha$, and IL-1RA) being the main triggers of inflammation. In linear discriminant analysis, MIS-C and KD profiles overlapped; however, a subgroup of MIS-C patients (MIS- $\mathrm{C}^{\text {plus }}$ ) differentiated from the remaining MIS-C patients in IFN- $\gamma$, IL-18, CM-CSF, RANTES, IP-10, IL-1 $\alpha$, and SDF-1 and incipient signs of macrophage activation syndrome. Circulating SARS-CoV-2 ICs were not detected in MIS-C patients. Our findings suggest a major role for IFN- $\gamma$ in the pathogenesis of MIS-C, which may be relevant for therapeutic management.
\end{abstract}

\section{Introduction}

Late in April 2020, a multisystem inflammatory syndrome temporally associated with SARS-CoV-2 was described in children (MIS-C) (1), occurring 4-6 weeks after the infectious peak (2-6). The diagnostic criteria for this entity were developed by health agencies based on the initial cases described (7-9), lacking specific biomarkers (7-10).

Authorship note: AES and JA contributed equally to this work. IJ and LA are co-senior authors.

Conflict of interest: The authors have declared that no conflict of interest exists. Copyright: (c) 2021, American Society for Clinical Investigation.

Submitted: September 28, 2020; Accepted: January 20, 2021; Published: March 15, 2021. Reference information: J Clin Invest. 2021;131(6):e144554.

https://doi.org/10.1172/JCl144554.
MIS-C shares common features with Kawasaki disease (KD) and patients were initially treated following recommendations for KD. However, there are notable differences, with the incidence of MIS-C being higher (2) and patients presenting with (a) older age, (b) increased gastrointestinal and neurological signs, (c) higher incidence of myocarditis and cardiac involvement, and (d) increased ferritin, leukopenia, lymphopenia, and thrombocytopenia $(2-5,11,12)$. Godfred-Cato et al. (13) recently identified 3 nonexclusive categories of patients within MIS-C: Class 1, with increased gastrointestinal and neurological manifestations, also named "true" or classic MIS-C (10); Class 2 or "acute COVID," most of them positive for SARS-CoV-2 by polymerase chain reaction (PCR) and negative by serology; and Class 3 or "KD-like" (10), with a phenotype similar to that of prepandemic KD. 
Table 1. Description of the study cohort: clinical data and SARS-CoV-2-related analytical data

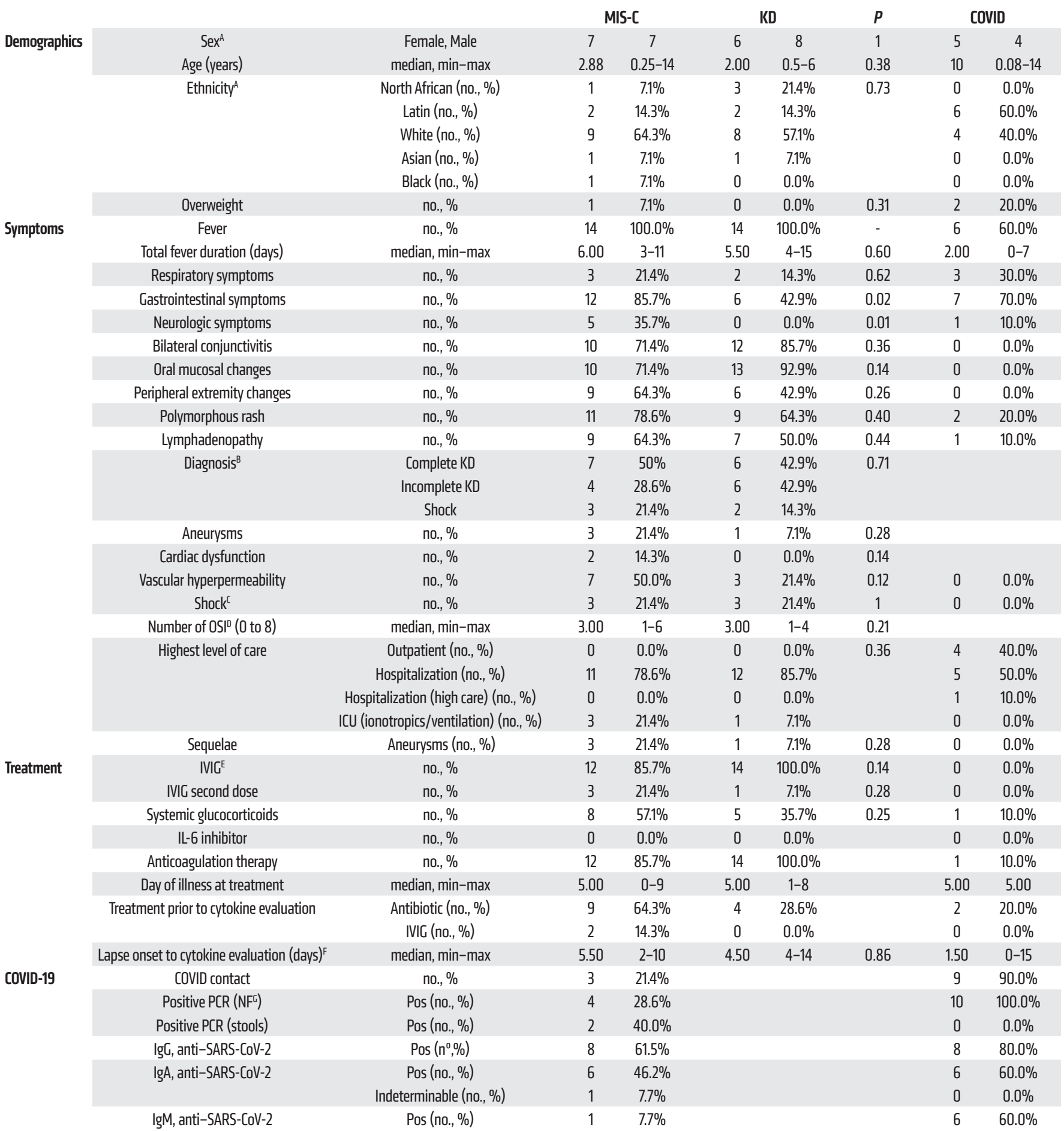

${ }^{A}$ Classified by the investigators. ${ }^{\mathrm{B}} 2017$ AHA criteria. ${ }^{C}$ Kanegaye Kawasaki shock criteria (45). ${ }^{\circ}$ Organ systems involvement. EIntravenous immunoglobulins. FDays between onset of disease and sample extraction. 'Nasopharyngeal. KD, pre-SARS-CoV-2 pandemic Kawasaki disease; MIS-C, multisystem inflammatory syndrome in children; COVID, pediatric patients with mild COVID-19 without MIS-C; IVIG, intravenous immunoglobulin; PCR, polymerase chain reaction; OSI, organ systems involvement; ICU, intensive care unit.

The underlying mechanism of MIS- $\mathrm{C}$ remains elusive. Currently, KD is categorized as a vasculitis (14) and it is considered to result from the exposure of a genetically predisposed child to an unidentified, possibly infectious agent. This interaction may generate an imbalance in the immune system, with increased Th1/Th17-related immunity and a Treg/Th17 imbalance. This 
Table 2. Cohort description: laboratory data

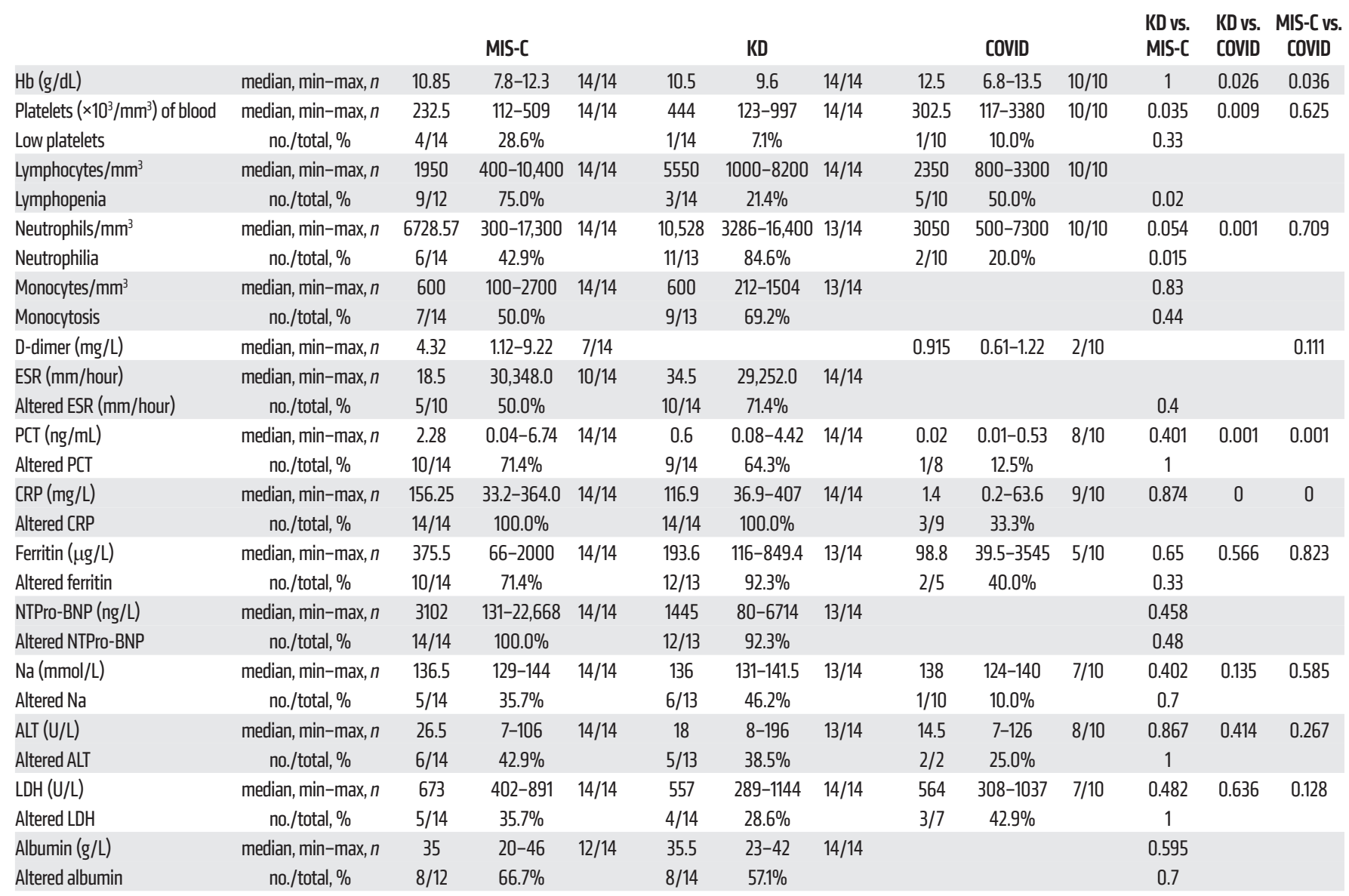

KD, pre-SARS-CoV-2 pandemic Kawasaki disease; MIS-C, multisystem inflammatory syndrome in children; COVID, pediatric patients with mild COVID-19 without MIS-C; ND, not determined; y, years; Hb, hemoglobin; ESR, erythrocyte sedimentation rate; PCT, procalcitonin; CRP, C-reactive protein; NTPro-BNP, $\mathrm{N}$-terminal pro-brain natriuretic peptide; ALT, alanine transaminase; LDH, lactate dehydrogenase; $\mathrm{Na}$, sodium.

would lead to increased production of inflammatory cytokines and chemokines such as TNF- $\alpha$, IL-1, IL-2, IL-6, IL-8, MCP-1, and GM-CSF, causing macrophage and neutrophil hyperactivation (15). SARS-CoV-2 is a single-stranded RNA virus (16) and infections caused by RNA viruses have been linked to KD (11). Type III hypersensitivity reactions may explain part of the pathogenesis of KD by immune complexes (ICs), as causal association has been reported $(11,17,18)$. Of note, deposited ICs were detected in the endothelium of an adult patient with SARS-CoV-2-related vasculitis 18 days after an infection confirmed by PCR (19).

We hypothesized that (a) cytokine profiles observed in MIS-C and prepandemic KD patients may be unique, and (b) SARSCoV-2-specific ICs may explain the immunopathology of MIS-C.

\section{Results}

Cohort description. Seventy-four children were studied: 14 with MIS-C (median age 2.9 years, range $0.3-14$ ); 14 with prepandemic KD (median age 2 years, range 0.5-6); 9 COVID patients positive for SARS-CoV-2 by nasopharyngeal reverse transcriptase PCR (RT-PCR) (mean age 10 years, range 0.1-14); and 37 healthy controls (HCs, median age 5 years, range 1-11; among the latter, 16 of $37 \mathrm{HC}$ patients had both serum and plasma samples collected in parallel, adding up to a total of $53 \mathrm{HC}$ samples). The clinical characteristics of 12 out of the 14 MIS-C patients of this cohort have previously been described by Pino et al. (6), showing increased gastrointestinal and neurological symptoms, increased lymphopenia and thrombopenia, and decreased neutrophilia, with statistically significant differences compared with KD patients (Tables 1 and 2 and Supplemental Tables 1 and 2; supplemental material available online with this article; https://doi.org/10.1172/JCI144554DS1). Eight of the 14 MIS-C patients had SARS-CoV-2-specific IgG, 6 of whom were also positive for IgA and none for IgM (Figure 1). MIS-C patients were treated according to recommendations for $\mathrm{KD}$ in the absence of specific recommendations at that time. The included COVID patients had a mild disease course; 7 out of 9 were positive for IgG.

Cytokine profiling. Cytokine profiling was performed in serum or plasma samples prior to immunomodulatory treatment (except in 2 patients who received immunoglobulins 2 days before blood extraction) and at a median time from disease onset of 5.50, 4.50, 

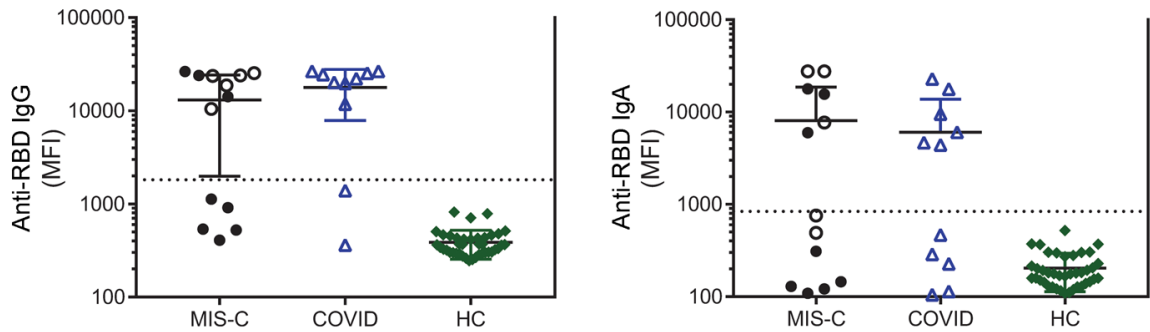

Figure 1. SARS-CoV-2-specific immunoglobulin detection. Determination of IgG, IgA, and IgM specific for the receptor-binding domain (RBD) of the SARS-CoV-2 spike protein. Open symbols represent patients with positive PCR in nasal swab or stools. Dotted lines represent threshold for positivity. MFI mean fluorescence intensity; MIS-C, multisystem inflammatory syndrome in children $(n=13)$; COVID, pediatric patients with mild COVID-19 disease without MIS-C $(n=9)$; HC, healthy controls ( $n=37$ [53 samples]).

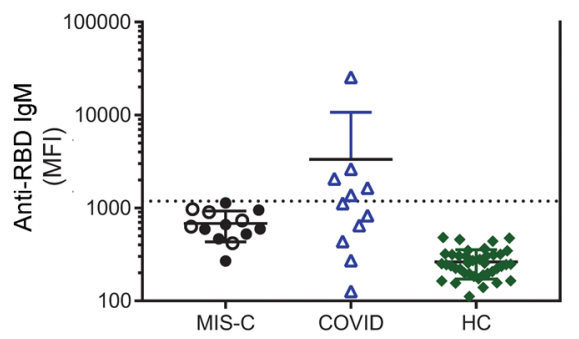

and 1.50 days for MIS-C, KD, and COVID patients, respectively. Plasma and serum cytokine levels were normalized to plasma and serum $\mathrm{HC}$ samples and transformed by $\log _{2}$ (fold change) ( $\left.\log _{2} \mathrm{FC}\right)$, to avoid sample-source-dependent differences (Supplementary Methods and Supplemental Figure 1) and were compared across groups (Supplemental Table 3 and Supplemental Figure 2).

In order to compare the 34-cytokine profile across groups, we performed an unsupervised multiparametric analysis through principal component analysis (PCA) (Figure 2A). In PCA, principal components (PCs) explain the maximum variance among all individuals. After PCA plotting of PC1 and PC2, HCs and mildCOVID patients were grouped in the same area, being still distinguishable, but apart from the MIS-C and KD groups, which partially overlapped. We identified 13 cytokines with a higher impact on the observed variance (Figure 2B). Comparison of cytokine levels between $\mathrm{KD} / \mathrm{MIS}-\mathrm{C}$ and $\mathrm{HC}$ showed statistically significant differences in all cytokines except IL-2, TNF- $\alpha$, MIP-1 $\alpha$, MIP-1 $\beta$, and IL-22 (Supplemental Table 3 and Supplemental Figure 2).

Although mild-COVID patients were grouped in the same area as HCs, they were not superimposed. Circulating cytokine levels of mild-COVID patients and HCs were statistically significantly different in 17 of 34 cytokines measured, including IFN- $\gamma$, IP-10, IL- $1 \alpha$, GM-CSF, GRO- $\alpha$, and SDF- 1 of the selected PCA cytokines (Figure 2B). However, their increase in mild-COVID patients was lower than that observed in MIS-C or KD patients.

We performed a supervised analysis through linear discriminant analysis (LDA) based on 13 selected PC1 and PC2 cytokines, to explore if they were sufficient to create a model that could distinguish between the different groups, in particular MIS-C and KD. LDA generated 3 canonical variants (Can1-Can3), explaining the maximum variability between groups; Can1 and Can2 allowed visual differentiation of $\mathrm{HC}$ and COVID from the other groups, but not KD from MIS-C (Figure 2C). Testing the model using the leaveone-out cross-validation was useful for distinguishing between $\mathrm{HC}$, COVID, and MIS-C+KD, but not between KD and MIS-C (Figure 3).

Visualization of Can2 versus Can3 showed that 5 patients with MIS-C (MIS-C $\mathrm{C}^{\text {plus }}$ ) presented a unique pattern (Figure 2D). Of the 13 cytokines selected to build the LDA model, IFN- $\gamma$, IL-18,
GM-CSF, RANTES, IP-10, IL-1 $\alpha$, and SDF-1 significantly differentiated MIS- $C^{\text {plus }}$ patients from the remaining MIS-C patients (Supplemental Table 4 and Figure 2E). The absolute values of these cytokines are shown in Figure 4. Specifically, the median circulating IFN- $\gamma$ levels in MIS-C ${ }^{\text {plus }}$ patients were $114.9 \mathrm{pg} / \mathrm{mL}$ (range 44.4-259.5 pg/mL; Figure 4), which was higher than in the remaining MIS-C patients (median of $10 \mathrm{pg} / \mathrm{mL}$ ), KD patients (median of $19.9 \mathrm{pg} / \mathrm{mL}$ ), and mild-COVID patients $(7.5 \mathrm{pg} / \mathrm{mL}$ ) (Figure 4 ). Although only $33 \%$ of all MIS-C patients were classified as MIS-C after leave-one-out cross-validation, $80 \%$ of MIS$\mathrm{C}^{\text {plus }}$ patients were classified as MIS-C (Figure 3).

MIS-C $\mathrm{C}^{\text {plus }}$ patients tended to have increased organ system involvement compared with MIS-C (median 5 [range 2-6] vs. 3 [range 1-6], respectively, $P=0.08$; Supplemental Table 5). MIS- $C^{\text {plus }}$ patients presented with increased neurological symptoms compared with the remaining MIS-C patients ( $80 \%$ vs. $11 \%, P=0.023$; Supplemental Table 5). SARS-CoV-2 RT-PCR testing in stool was performed in $80 \%$ of MIS-C ${ }^{\text {plus }}$ patients compared with only 2 of the 9 remaining MIS-C patients, suggesting an increased severity of gastrointestinal symptoms. In addition, SARS-CoV-2-specific IgA was positive or indeterminate in $80 \%$ of MIS-C $\mathrm{C}^{\text {plus }}$ patients compared with $37.5 \%$ of the remaining MIS-C patients $(P=0.21)$. Although not reaching statistical significance, MIS- $C^{\text {plus }}$ patients displayed lower platelet levels (median of 170,000 vs. 373,000 platelets $\left./ \mathrm{mm}^{3}, P=0.08\right)$ and a higher frequency of altered procalcitonin $(100 \%$ vs. $55.6 \%, P=0.08)$ and ferritin values $(100 \%$ vs. $55.6 \%, P=0.08$ ) (Supplemental Table 6).

Detection of SARS-CoV-2 ICs. SARS-CoV-2 ICs were determined by quantifying SARS-CoV-2 RNA before and after immunoprecipitation of IgA and IgG, chosen because SARS-CoV-2-specific IgG and IgA were previously reported in MIS-C patients (Figure 1 and ref. 20) and because of their reported role in KD IC-mediated pathogenesis (17). We performed SARS-CoV-2 real-time RT-PCR in (a) pretreated serum, (b) IgG-immunoprecipitated serum, (c) IgA-immunoprecipitated serum, and (d) IgG- and IgA-free serum in MIS-C patients and in 1 positive and 1 negative control. We did not detect circulating SARS-CoV-2 RNA nor SARS-CoV-2 ICs in any MIS-C patients (Supplemental Figure 3). 

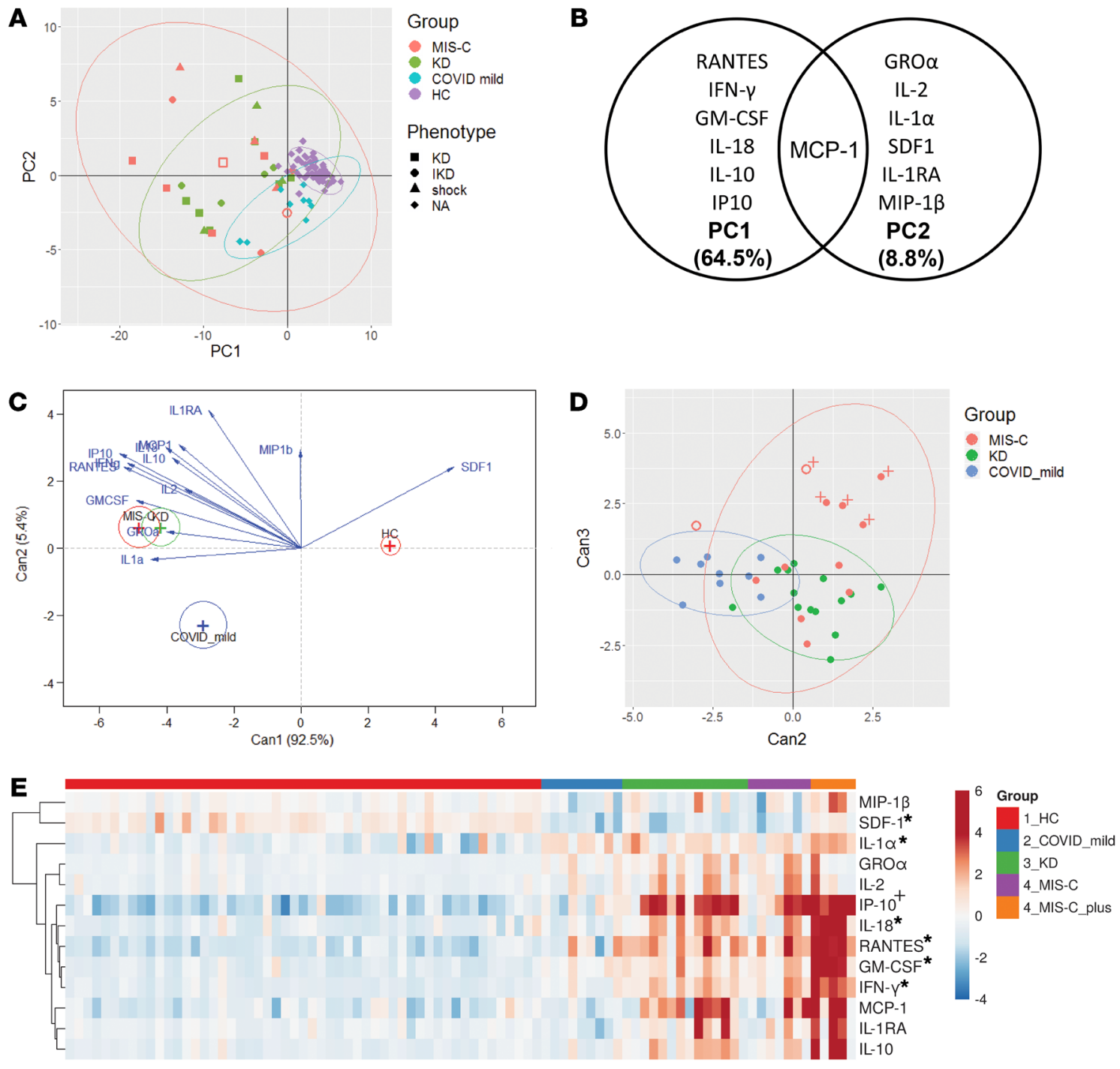

Figure 2. Cytokine profile of the different groups. Levels of 34 circulating cytokines were evaluated in serum or plasma. (A) Principal component analysis: every data point represents one individual. (B) Total variance explained by principal component 1 (PC1) and PC2 and variables with higher scores. (C) Representation of canonical variants (Can1 and -2) of the linear discriminant analysis (LDA) built with the cytokines represented in B. (D) Representation of Can2 and Can3 of the LDA (HCs have been removed from the plot, not from the analysis). A " + " indicates subgroup of MIS-C patients differentiated from other MIS-C and KD patients (MIS-Cplus). Open symbols represent patients who previously received immunoglobulin treatment. (E) Heatmap representing the selected cytokines (represented in panel B). Every column represents a patient. An asterisk $\left(^{*}\right)$ identifies the cytokines with statically significant differ-

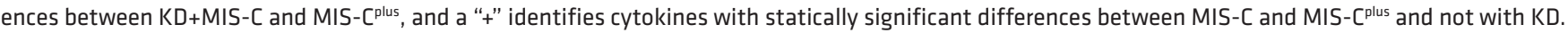
Color gradient represents the $z$ score. KD, pre-SARS-CoV-2 pandemic Kawasaki disease $(n=14)$; MIS-C, multisystem inflammatory syndrome in children $(n=13)$; COVID, pediatric patients with mild COVID-19 disease without MIS-C $(n=9)$; HC, healthy controls $(n=37$ [53 samples]).

\section{Discussion}

SARS-CoV-2 seemed to have spared children until the appearance of MIS-C in pediatric patients, which at first was classified as a "Kawasaki-like" manifestation of the infection (1). In order to better understand the similarities and differences between these 2 entities, we evaluated circulating cytokine levels in pediatric patients with MIS-C, prepandemic KD, and mild COVID, and the presence of SARS-CoV-2 ICs in MIS-C patients. Our findings suggest that (a) IFN- $\gamma$ and inflammatory monocytes are the main drivers of inflammation in MIS-C patients and (b) MIS-C patients can be subdivided into 2 groups: 1 similar to KD in terms of clinical and cytokine profiles (described as Class 3, ref. 13; or KD-like, ref. 10), and 1 described as Class 1 (13) ("true" or classic MIS-C; ref. 10), with more severe inflammation (especially IFN- $\gamma$-related) and increased neurological and gastrointestinal manifestations. Circulating SARS-CoV-2 ICs could not be identified in MIS-C patients.

Clinical and routine laboratory parameters of MIS-C patients as well as SARS-CoV-2-specific serology results were in accordance with other published reports, in which SARS-CoV-2specific IgG antibodies have been observed before day 10 of symptom onset $(2-4,20-22)$. Circulating cytokine determination included 34 cytokines and chemokines, covering most of the cytokines described as being elevated in MIS-C patients $(5,21,23-25)$. After PCA, we selected 13 cytokines that explained most of the observed variance across individuals. Selected cytokines in PC1 


\begin{tabular}{|c|cccc|}
\cline { 2 - 5 } \multicolumn{1}{c|}{} & MIS-C & KD & COVID & HC \\
\hline $\begin{array}{c}\text { MIS-C } \\
\text { (MIS-C }\end{array}$ & $\begin{array}{c}33.33 \% \\
(80 \%)\end{array}$ & $\begin{array}{c}50.00 \% \\
(20 \%)\end{array}$ & $\begin{array}{c}16.67 \% \\
(0 \%)\end{array}$ & $\begin{array}{c}0.00 \% \\
(0 \%)\end{array}$ \\
KD & $42.86 \%$ & $42.86 \%$ & $14.29 \%$ & $0.00 \%$ \\
COVID & $11.11 \%$ & $22.22 \%$ & $66.67 \%$ & $0.00 \%$ \\
HC & $0.00 \%$ & $0.00 \%$ & $0.00 \%$ & $100.00 \%$ \\
\hline
\end{tabular}

(64.5\% of the variance) were enriched in IFN- $\gamma$-related cytokines, while those in PC2 (8.8\% of the variance) were enriched in IL-1 and inflammatory monocyte pathways (Figure 2B), suggesting a principal role for these cytokines in the inflammation observed in $\mathrm{KD}$ and MIS-C. Activation of inflammatory monocytes (CD16 ${ }^{+}$ has already been described in MIS-C patients $(21,23)$ as well as in severe-SARS-CoV-2 adult patients (18). In addition, a moderate increase in circulating cytokine levels observed in mild-COVID patients was in accordance with previous reports $(21,23)$.

Previous reports have also evaluated circulating-cytokine profiles in MIS-C patients $(5,21,23,25,26), 2$ of which included KD patients $(5,25)$. Although a similar approach was used, the cohorts are not fully comparable. There were differences in the definition of HCs and COVID patients, and the time from disease onset to cytokine testing was only specified in 1 study (23); thus, it is unknown whether testing was prior to immunomodulatory treatment. Despite these differences, reported evidence shows that MIS-C patients display an increase in inflammatory cytokines including IFN- $\gamma(24)$, IL-10 (23-25), IL-6 (5, 24, 25), IL-8 (23, 24), CXCL10 (21, 23, 27), MIP- $1 \alpha$, MIP-1 $\beta$ (21), TNF- $\alpha(23,24)$, and IL-17 $(22,28)$, in MIS-C patients compared with HCs and other pediatric COVID patients. These results are in accordance with our observations (Supplemental Figure 2 and Supplemental Table 3). In addition, CXCL9 (25) and IL-2RA $(23,25)$ were also found to be increased in MIS-C patients.

Consiglio et al. (5) compared MIS-C patients from Italy and Sweden with prepandemic Italian KD patients. They performed PCA of 180 proteins in plasma, finding that a subgroup of MIS-C patients overlapped with KD patients and another clearly differentiated. They found an especially large increase in IL-17 in KD patients in comparison with HC and COVID patients, which was not observed in MIS-C patients. We and others $(21,23)$ have observed a marked increase in circulating IL-17 levels in KD patients but also in MIS-C patients, though to a lesser extent in the latter (1.15 vs. 1.50 median $\log _{2} \mathrm{FC}$ in MIS-C and KD patients, respectively, compared with $\mathrm{HC}$; $6.84 \mathrm{pg} / \mathrm{mL}$ vs. $13.63 \mathrm{pg} / \mathrm{mL}$ vs. $1.40 \mathrm{pg} / \mathrm{mL}$ median quantitative levels in MIS-C, KD, and HC, respectively; Supplemental Table 3 and Supplemental Figure 4). These differences may be ascribed to a different behavior of the Swedish cohort, taking into account the medRxiv version of the paper (27).

The marked increase in circulating levels of IFN- $\gamma$, IL-18, and IP-10 in MIS-C $\mathrm{C}^{\text {plus }}$ patients in comparison with other MIS-C and KD patients led to suspicion of a possible relationship with macrophage activation syndrome (MAS) (29-31). Indeed, the presence of MAS was plausible in 1 patient (MIS-C $C^{\text {plus }}$ ID 11; Supple-
Figure 3. Leave-one-out cross-validation of linear discriminant analysis model. Rows represent the preclassified groups and columns the predicted categories after leave-one-out cross-validation. KD, pre-SARS-CoV-2 pandemic Kawasaki disease $(n=14)$; MIS-C, multisystem inflammatory syndrome in children $(n=13)$; COVID, pediatric patients with mild COVID-19 disease without MIS-C $(n=9) ; \mathrm{HC}$, healthy controls ( $n=31$ [53 samples]).

mental Table 2). These cytokines are also known to be increased in severe-COVID patients $(18,32)$. Elevation of circulating IFN- $\gamma$ is a hallmark of hemophagocytic syndromes (hemophagocytic lymphohistiocytosis, HLH) including MAS, and its determination in serum in different studies has shown varying results. IFN- $\gamma$ is normally undetectable in HCs (28). Increased levels of IFN- $\gamma$ have been reported in viral infections (mean of $18.9 \mathrm{pg} / \mathrm{mL}$, range 7.7$33.4 \mathrm{pg} / \mathrm{mL}$; ref. 33) and particularly high serum levels are detected in patients with suspected primary HLH (fulfilling HLH-2004 diagnostic criteria [ref. 34], below 10 years of age, with or without confirmed genetic diagnosis) (mean of $1807 \mathrm{pg} / \mathrm{mL}$ (range 147.6 to $>5000 \mathrm{pg} / \mathrm{mL}$; ref. 33) and $905 \mathrm{pg} / \mathrm{mL}$ (95\% CI 530.7-1280.6 pg/ $\mathrm{mL}$; ref. 35). Patients with MAS associated with systemic juvenile idiopathic arthritis showed mean serum IFN- $\gamma$ levels of $15.4 \mathrm{pg} /$ $\mathrm{mL}$ (5.1-52.6 pg/mL IQR), although using a different quantification technique in comparison with the other 2 studies (36). Thus, MIS-C $C^{\text {plus }}$ median circulating IFN- $\gamma$ levels were higher than in viral infections and MAS, but lower than in suspected primary HLH, therefore suggesting that circulating IFN- $\gamma$ levels in MIS-C $C^{\text {plus }}$ cannot only be ascribed to a viral infection but rather may represent a mechanism of disease.

Negative detection of ICs does not rule out their possible role in the immunopathogenesis of MIS-C and this might be explained by 2 hypotheses: (a) the ICs may already be deposited in the endothelium and thus are not detectable in serum/plasma, or (b) the pathogenesis may be mediated by direct infection of the endothelium. ICs have been described in vascular tissue of a SARS-CoV-2 patient with vasculitis (19) and SARS-CoV-2 viral particles have been observed inside the endothelium in pediatric patients with SARS-CoV-2-related chilblains (37). The absence of vascular tissue biopsies limits further exploration of these hypotheses.

Our study has both strengths and limitations. It is of note that most samples were collected within the first week after the beginning of the symptoms and prior to any immunomodulatory treatment except in only 2 of 74 children, who were still clinically active and presented with an inflammatory profile. In addition, the cytokine profile was normalized to plasma and serum of age-matched pediatric HCs, enabling a more robust comparison between different groups and interpretation of results. On the other hand, the cohorts were modest in size, but large enough to observe differences. Also, we had limited access to different types of samples, including peripheral blood mononuclear cells and tissue biopsies, that could have been useful for evaluation of $\mathrm{T}$ cell immunity and monocyte phenotyping. 

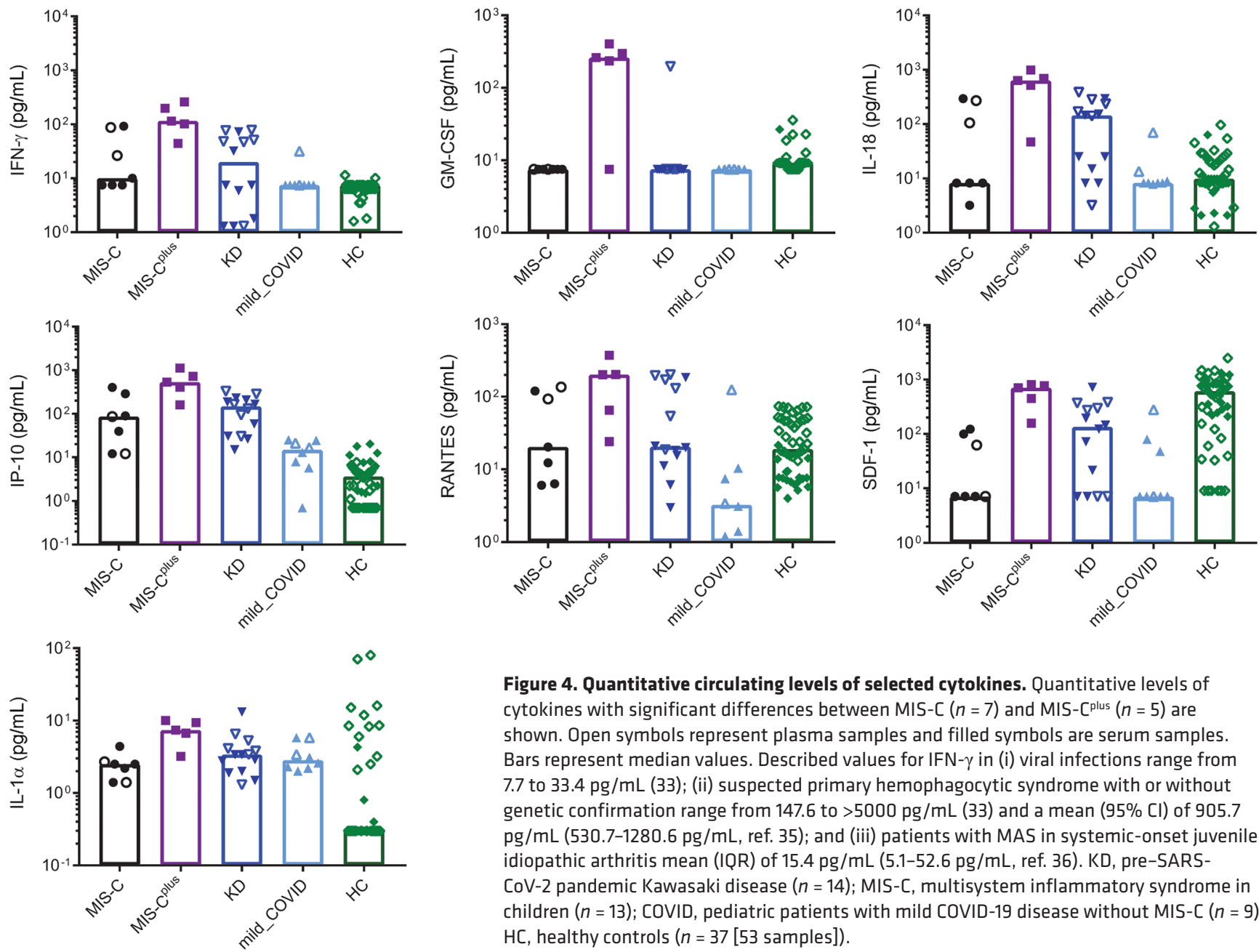

\begin{abstract}
Figure 4. Quantitative circulating levels of selected cytokines. Quantitative levels of cytokines with significant differences between MIS-C $(n=7)$ and MIS-Cplus $(n=5)$ are shown. Open symbols represent plasma samples and filled symbols are serum samples. Bars represent median values. Described values for IFN- $\gamma$ in (i) viral infections range from 7.7 to $33.4 \mathrm{pg} / \mathrm{mL}$ (33); (ii) suspected primary hemophagocytic syndrome with or without genetic confirmation range from 147.6 to $>5000 \mathrm{pg} / \mathrm{mL}$ (33) and a mean $(95 \% \mathrm{Cl})$ of 905.7 $\mathrm{pg} / \mathrm{mL}$ (530.7-1280.6 pg/mL, ref. 35); and (iii) patients with MAS in systemic-onset juvenile idiopathic arthritis mean (IQR) of $15.4 \mathrm{pg} / \mathrm{mL}$ (5.1-52.6 pg/mL, ref. 36). KD, pre-SARSCoV-2 pandemic Kawasaki disease $(n=14)$; MIS-C, multisystem inflammatory syndrome in children ( $n=13)$; COVID, pediatric patients with mild COVID-19 disease without MIS-C $(n=9)$; $\mathrm{HC}$, healthy controls ( $n=37$ [53 samples]).
\end{abstract}

In summary, our results replicate the routine-clinical laboratory differences observed between $\mathrm{KD}$ and MIS-C $(2,5,25)$. In our cohort, IFN- $\gamma$-related cytokines and cytokines related to IL-1 and inflammatory monocytes were the main contributors to acute inflammation both in MIS-C and KD patients, which mostly overlapped. A subgroup of MIS-C patients (MIS-C $C^{\text {plus }}$ ) showed a more pronounced increase in IFN- $\gamma$-related cytokines and a trend toward a more severe multisystemic disorder, resembling the Class 1 (13) ("true" or classic MIS-C; ref. 10) (MIS-C ${ }^{\text {plus }}$ patients) versus Class 3 or "KD-like" (ref. 10; remaining MIS-C patients) classification of Godfred-Cato et al. (13). We speculate that the differential gastrointestinal involvement of SARS-CoV-2 infection may have contributed to the differential cytokine profile observed in MIS-C $\mathrm{C}^{\text {plus }}$ compared with KD patients. Our findings may be useful for the development of targeted treatments to optimize patient management and, ultimately, might reduce the estimated mortality of $2 \%$ in patients with MIS-C (4).

\section{Methods}

From April 23 (4 weeks after the exponential increase of COVID-19 cases in our area and schools closed) to June 5, 2020, 14 patients with MIS-C (according to WHO, ref. 7, or Royal College criteria, refs. 8, 9) pediatric patients positive for SARS-CoV-2 by PCR without MIS-C
(COVID) and 37 pediatric HCs negative for SARS-CoV-2 by PCR and serology were recruited. Fourteen patients with prepandemic KD, according to AHA criteria (38), were also recruited between 2016 and 2019. Patient definition and inclusion criteria are defined in Supplemental Methods.

This was a cross-sectional study. Patients' blood samples were drawn between day 1 and day 10 of disease evolution (with the exception of $1 \mathrm{KD}$ patient in whom disease onset had been 14 days but who still presented with increased C-reactive protein levels) for routine laboratory analysis, quantification of 34 different circulating cytokines and evaluation of the presence of circulating SARS-CoV-2 ICs (only in patients with MIS-C). Sampling was performed before the administration of immunomodulatory treatment (intravenous immunoglobulin [IVIG], steroids, tocilizumab, anakinra) in all but 2 patients who received IVIG 2 days before the extraction. None of the patients had preexisting conditions, except for 1 COVID patient in remission of an acute lymphocytic leukemia after stem cell transplantation in 2017.

\section{SARS-CoV-2 infection determination}

Real-time RT-PCR for nasopharyngeal and stool samples. Viral RNA extraction was performed with NuclisENS easyMAG (BioMerieux Laboratories) or MagMAX Viral/Pathogen Nucleic Acid Isolation Kit (Thermo Fisher Scientific) following the manufacturers' instruc- 
tions. RT-PCR was performed with the COVID-19 Plus RealAmp Kit (Genefinder Laboratories) for detection of the RdRp, E, and $\mathrm{N}$ viral genes and the human RNase P gene as internal control.

\section{SARS-CoV-2 serology}

IgG, IgA, and IgM antibodies specific for SARS-CoV-2 were determined using a Luminex system against the receptor-binding domain of the spike glycoprotein of SARS-CoV-2, as reported previously (39). Briefly, $10 \mu \mathrm{L}$ of plasma was incubated with antigen-coupled beads for 2 hours at room temperature with agitation. Plates were then washed 3 times and incubated with biotinylated secondary antibody (IgM, IgA, or IgG; Sigma-Aldrich) for 45 minutes at room temperature with agitation. Plates were washed 3 times and streptavidin-R-phycoerythrin (Sigma-Aldrich) was added for 30 minutes at room temperature with agitation. Plates were washed 3 times and beads were resuspended in phosphate-buffered saline (PBS) (Roche Diagnostics). Plates were read using a Luminex xMAP 100 analyzer.

\section{Cytokine quantification}

Cytokine determination was assessed with the Luminex System (Procartaplex, Thermo Fisher Scientific) performed in serum or plasma of patients the following the manufacturer's instructions. The cytokines detected included eotaxin/CCL11, GM-CSF, GRO- $\alpha$ /CXCL1, IFN- $\alpha$, IFN- $\gamma$, IL-1 $\beta$, IL-1 $\alpha$, IL-1RA, IL-2, IL-4, IL-5, IL-6, IL-7, IL-8/CXCL8, IL-9, IL-10, IL-12 p70, IL-13, IL-15, IL-17A, IL-18, IL-21, IL-22, IL-23, IL-27, IL-31, IP-10/CXCL10, MCP-1/CCL2, MIP-1 $\alpha / C C L 3$, MIP-1 $\beta$ / CCL4, RANTES/CCL5, SDF-1/CXCL12, TNF- $\alpha$, and TNF- $\beta / L T A$. Plates were read using a Luminex xMAP 100 analyzer.

\section{SARS-CoV-2 IC evaluation}

Specific viral ICs against SARS-CoV-2 were detected by adapting a previous protocol used to detect hepatitis C virus ICs $(40,41)$. This consisted of the immunoprecipitation of IgG or IgA followed by determination of viral load with quantitative PCR. Specifically, for each sample, $25 \mu \mathrm{L}$ of $10 \mathrm{mg} / \mathrm{mL}$ streptavidin magnetic beads (Thermo Fisher Scientific) was incubated with $5 \mu \mathrm{g}$ of IgG-biotin (Sigma-Aldrich) or IgA-biotin (Sigma-Aldrich) for each sample for 30 minutes at room temperature with orbital agitation. After 2 washes with PBS containing $0.05 \%$ bovine serum albumin (BSA) (Sigma-Aldrich), the magnetic beads were resuspended to the original volume with PBSBSA. Twenty-five microliters of magnetic beads coupled with IgG were incubated with $100 \mu \mathrm{L}$ of patient serum for 30 minutes at room temperature with orbital agitation. Magnetic separation between the beads and the supernatant was performed. The process was repeated with resulting streptavidin- and IgA-coupled magnetic beads. SARSCoV-2 virus was measured in pretreated serum, the IgG-immunoprecipitated fraction, the IgA-immunoprecipitated fraction, and supernatant fraction by quantitative PCR.

For the detection of nucleic acid from SARS-CoV-2 in serum samples, RNA was isolated with the MagMAX Viral/Pathogen Nucleic Acid Isolation Kit (Thermo Fisher Scientific), following the manufacturer's instructions. Multiplex real-time RT-PCR (TaqPath COVID-19 CE-IVD RT-PCR Kit, Thermo Fisher Scientific) was performed in an Applied Biosystems 7500 Fast Real-Time PCR System (Thermo Fisher Scientific) to detect the genes encoding SARS-CoV-2 ORF1ab, N protein, and S protein. An internal control (MS2 phage control) was included before RNA extraction. The assay included a positive control, consisting of a sample with positive SARS-CoV-2 and positive SARSCoV-2-specific antibodies and a negative control, consisting on a sample negative for SARS-CoV-2 and SARS-CoV-2-specific antibodies.

\section{Routine hematological and biochemical analysis}

A broad battery of routine laboratory parameters including hemogram, erythrocyte sedimentation rate (ESR, $\mathrm{mm} /$ hour), D-dimer, procalcitonin (PCT), C-reactive protein (CRP), ferritin, $\mathrm{N}$-terminal pro-brain natriuretic peptide (NTPro-BNP), sodium, lactate dehydrogenase $(\mathrm{LDH})$, and albumin were studied within the routine hospital care.

\section{Statistics}

For the analysis of clinical data, the Mann-Whitney test was performed to compare numerical variables between 2 groups and the $\chi^{2}$ test was used to compare categorical variables between 2 groups.

For the analysis of cytokine levels, we used relative changes in the fluorescence intensities, as recommended previously (42). Because we had both plasma and serum samples from patients (Supplemental Table 7) and the matrix from plasma and serum may affect the results (42), data from serum samples were normalized to the mean of serum samples from $\mathrm{HCs}$, and data from plasma samples were normalized to the mean of plasma samples from HCs to minimize this effect. After normalization to $\mathrm{HCs}, \log _{2} \mathrm{FC}$ of all the samples was compared to the mean of the HC group (ref. 22 and Supplemental Figure 1).

For PCA we used single value decomposition without data scaling because data were already normalized. PCA and heatmap analysis were performed using the online tool ClustVis (43). LDA was performed on the normalized data using the $\mathrm{R}$ package candisc (discriminant and canonical correlation analysis, R package version 0.8; ref. 44).

The Kruskal-Wallis test was performed for all the cytokines, using Bonferroni's correction for comparisons of all the samples. Pair-wise comparisons using the Mann-Whitney test were only performed when the Kruskal-Wallis test was significant after Bonferroni's correction. Mann-Whitney test results were corrected for multiple comparisons (4 groups, 6 comparisons) with Bonferroni's correction. Statistical test results were considered significant when $P$ was less than 0.05 . To compare the circulating cytokine levels of MIS-C $C^{\text {plus }}$ patients with the remaining MIS-C and KD patients, Bonferroni's correction was not performed due to sample size limitation as previously reported (24). Taking into account the sample size, changes reported as statistically significant should be interpreted as indicative of the direction of change in biological signals.

The statistical analyses and graphical representation of the data were done with SPSS 22 (IBM), Prism 8 (GraphPad Software), the online tool ClustVis (43), and R 3.6 (Foundation for Statistical Computing).

\section{Study approval}

This study was carried out in accordance with the recommendations of the Ley General de Sanidad (April 25, 1986) Art. 10. The protocol was approved by the Ethics Committee of Hospital Sant Joan de Déu (PIC-58-60). Parents and children over the age of 12 years signed informed consent or assent, in compliance with current legislation.

\section{Author contributions}

LA, IJ, AES, JA, MJ, VF, and CF conceptualized and designed the research. AES performed immunologic studies and prepared the figures. AV, EGR, JC, and MJ performed SARS-CoV-2 detection. 
LA, AES, RMPR, and IJ analyzed and interpreted data. RMPR, JSM, VF, CF, MRB, JSDT, MGA, JMM, SR, CL, MFDS, CJ, CMA, and IJ provided patient material and clinical data and participated in discussion of the results. AES, JA, RMPR, SR, ANJ, DC, IJ, and LA discussed the integration of the data. AES and LA wrote the manuscript. AES and JA share co-first authorship because AES was responsible for the immunologic studies, while JA was in charge of patient recruitment and clinical evaluation; AES is first coauthor because of her role in writing the manuscript. All authors have read the manuscript, contributed to manuscript revision, and approved the submitted version.

\section{Acknowledgments}

The authors especially thank the participants of the study and all the people involved in the care of the patients. We are indebted to the "Biobanc de l'Hospital Infantil Sant Joan de Déu per a la Investigació" integrated in the Spanish Biobank Network of ISCIII for the sample and data procurement. This study was supported by projects PI15/01094, PFIS0200 (AC16/00025),
PI18/00223, and FI19/00208 (to LA), integrated in the Plan Nacional de I+D+I and cofinanced by the ISCIII - Subdirección General de Evaluación y Formento de la Investigación Sanitaria -FEDER;PERIS, Departament de Salut, Generalitat de Catalunya (SLT006/17/00199 to LA); 2017 Leonardo Grant for Researchers and Cultural Creators, BBVA Foundation (to LA); 2017 Beca de Investigación de la SEICAP (to LA); a WINDBIOME grant from the Daniel Bravo Foundation; and an 018 SERPE grant from Socedad Española de Reumatología Pediátrica (SERPE). This work was also partially funded by Stavros Niarchos Foundation (SNF), Banco Santander, and other private donors of "Kids Corona platform" from Hospital Sant Joan de Déu and by CELLNEX TELECOM (5234-20/CPO42837).

Address correspondence to: Laia Alsina or to Ana EsteveSole, Hospital Sant Joan de Déu, Avinguda Sant Joan de Déu, 2, Esplugues del Llobregat, Barcelona 08950, Spain. Phone: 34.932.80.4000, ext.71712; Email: lalsina@sjdhospitalbarcelona. org (LA); aesteves@fsjd.org (AES).
1. Riphagen S, et al. Hyperinflammatory shock in children during COVID-19 pandemic. Lancet. 2020;395(10237):1607-1608.

2. Verdoni L, et al. An outbreak of severe Kawasaki-like disease at the Italian epicentre of the SARS-CoV-2 epidemic: an observational cohort study. Lancet. 2020;6736(20):1-1778.

3. European Centre for Disease Prevention and Control. Paediatric Inflammatory Multisystem Syndrome and SARS-CoV-2 Infection in Children. Accessed February 3, 2021. https://www.ecdc. europa.eu/sites/default/files/documents/covid19-risk-assessment-paediatric-inflammatorymultisystem-syndrome-15-May-2020.pdf.

4. Feldstein LR, et al. Multisystem inflammatory syndrome in US children and adolescents. N Engl J Med. 2020;383(4):334-346.

5. Consiglio CR, et al. The immunology of multisystem inflammatory syndrome in children with COVID-19. Cell. 2020;183(4):968-981.

6. Pino R, et al. Correspondence on: 'Paediatric multisystem inflammatory syndrome temporally associated with SARS-CoV-2 mimicking Kawasaki disease (Kawa-COVID-19): a multicentre cohort' by Pouletty, et al. [published online August 05, 2020]. Ann Rheum Dis. http://dx.doi. org/10.1136/annrheumdis-2020-218538.

7. World Health Organization. Multisystem inflammatory syndrome in children and adolescents temporally related to COVID-19. Accessed February 3, 2020. https://www.who.int/newsroom/commentaries/detail/multisysteminflammatory-syndrome-in-children-andadolescents-with-covid-19.

8. Royal College of Paediatrics and Child Health. Guidance: Paediatric Multisystem Inflammatory Syndrome Temporally Associated With COVID-19. https://www.rcpch.ac.uk/sites/ default/files/2020-05/COVID-19-Paediatric-multisystem-\%20inflammatory\%20 syndrome-20200501.pdf. Updated September, 2020. Accessed February 3, 2020.

9. Morris SB, et al. Multisystem inflammatory syndrome in children (MIS-C) associated with Coronavirus Disease 2019. Clinical Outreach and
Communication Activity webinar. May 21, 2020. Accessed February 3, 2021. https://emergency.cdc. gov/coca/calls/2020/callinfo_051920.asp.

10. Rowley $\mathrm{AH}$, et al. Immune pathogenesis of COVID-19-related multisystem inflammatory syndrome in children (MIS-C). JClin Invest. 2020;130(11):5619-5621.

11. Burns JC, Glodé MP. Kawasaki syndrome. Lancet. 2004;364(9433):533-544.

12. Cheung EW, et al. Multisystem inflammatory syndrome related to COVID-19 in previously healthy children and adolescents in New York City. JAMA. 2020;324(3):294-296.

13. Godfred-Cato S, et al. COVID-19-associated multisystem inflammatory syndrome in children - United States, March-July 2020. MMWR Morb Mortal Wkly Rep. 2020;69(32):1074-1080.

14. Ozen S. EULAR/PReS endorsed consensus criteria for the classification of childhood vasculitides. Ann Rheum Dis. 2005;65(7):936-941.

15. Takahashi K, et al. Update on etio and immunopathogenesis of Kawasaki disease. Curr Opin Rheumatol. 2014;26(1):31-36.

16. Perez-Toledo M, et al. Serology confirms SARSCoV-2 infection in PCR-negative children presenting with Paediatric Inflammatory Multi-System Syndrome [preprint]. https://doi.org/10.1101/2020 .06.05.20123117. Posted on medRxiv June 7, 2020.

17. Menikou S, et al. Kawasaki disease: the role of immune complexes revisited. Front Immunol. 2019;10:1156

18. Vabret N, et al. Immunology of COVID19: current state of the science. Immunity. 2020;52(6):910-941.

19. Roncati L, et al. Type 3 hypersensitivity in COVID-19 vasculitis. Clin Immunol. 2020;217:108487.

20. Weisberg SP, et al. Distinct antibody responses to SARS-CoV-2 in children and adults across the COVID-19 clinical spectrum. Nat Immunol. 2020;22(1):25-31.

21. Gruber CN, et al. Mapping systemic inflammation and antibody responses in multisystem inflammatory syndrome in children (MIS-C). Cell. 2020;183(4):982-995.
22. Jin $\mathrm{Y}$, et al. Diagnostic value and dynamic variance of serum antibody in coronavirus disease 2019. Int J Infect Dis. 2020;94:49-52.

23. Carter MJ, et al. Peripheral immunophenotypes in children with multisystem inflammatory syndrome associated with SARS-CoV-2 infection. Nat Med. 2020;26(11):1701-1707.

24. Diorio C, et al. Multisystem inflammatory syndrome in children and COVID-19 are distinct presentations of SARS-CoV-2. JClin Invest. 2020;130(11):5967-5975

25. Lee PY, et al. Distinct clinical and immunological features of SARS-COV-2-induced multisystem inflammatory syndrome in children. J Clin Invest. 2020;130(11):5942-5950.

26. Anderson EM, et al. SARS-CoV-2 antibody responses in children with MIS-C and mild and severe COVID-19 [published online December 2, 2020]. JPediatric Infect Dis Soc. https://doi. org/10.1093/jpids/piaa161.

27. Consiglio CR, et al. The immunology of multisystem inflammatory syndrome in children with COVID-19. Cell. 2020;183(4):968-981.

28. Fieschi C, et al. High levels of interferon gamma in the plasma of children with complete interferon gamma receptor deficiency. Pediatrics. 2001;107(4):E48.

29. Crayne CB, et al. The immunology of macrophage activation syndrome. Front Immunol. 2019;10:119.

30. Jinkawa A, et al. Cytokine profile of macrophage activation syndrome associated with Kawasaki disease. Cytokine. 2019;119:52-56.

31. Lee WI, et al. Immune defects in active mycobacterial diseases in patients with primary immunodeficiency diseases (PIDs). J Formos Med Assoc. 2011;110(12):750-758.

32. Huang K-J, et al. An interferon-gamma-related cytokine storm in SARS patients. J Med Virol. 2005;75(2):185-194.

33. Tang $\mathrm{Y}$, et al. Early diagnostic and prognostic significance of a specific Th1/Th2 cytokine pattern in children with haemophagocytic syndrome. $\mathrm{Br} J$ Haematol. 2008;143(1):84-91.

34. Henter JI, et al. HLH-2004: diagnostic and 


\section{RESEARCH ARTICLE}

therapeutic guidelines for hemophagocytic lymphohistiocytosis. Pediatr Blood Cancer. 2007;48(2):124-131.

35. Chen Y, et al. Comparison of Th1/Th2 cytokine profiles between primary and secondary haemophagocytic lymphohistiocytosis. Ital J Pediatr. 2016;42(1):50.

36. Bracaglia $\mathrm{C}$, et al. Elevated circulating levels of interferon- $\gamma$ and interferon- $\gamma$-induced chemokines characterize patients with macrophage activation syndrome complicating systemic juvenile idiopathic arthritis. Ann Rheum Dis. 2017;76(1):166-172.

37. Colmenero I, et al. SARS-CoV-2 endothelial infection causes COVID-19 chilblains: histopathological, immunohistochemical and ultra- structural study of 7 paediatric cases. Br J Dermatol. 2020;183(4):729-737.

38. McCrindle BW, et al. Diagnosis, treatment, and long-term management of kawasaki disease: a scientific statement for health professionals from the American Heart Association. Circulation. 2017;135(17):e927-e999.

39. Garcia-Basteiro AL, et al. Seroprevalence of antibodies against SARS-CoV-2 among health care workers in a large Spanish reference hospital. Nat Commun. 2020;11(1):3500.

40. Morita T, et al. Detection of hepatitis C virus RNA in circulating immune complexes by RT-PCR. Hepatogastroenterology. 1996;43(9):582-585.

41. Aiyama T, et al. Sequence analysis of hypervariable region of hepatitis $\mathrm{C}$ virus ( $\mathrm{HeV}$ ) associated with immune complex in patients with chronic HCV infection. J Infect Dis. 1996;174(6):1316-1319.

42. Rosenberg-Hasson Y, et al. Effects of serum and plasma matrices on multiplex immunoassays. Immunol Res. 2014;58(2-3):224-233.

43. Metsalu T, Vilo J. ClustVis: a web tool for visualizing clustering of multivariate data using Principal Component Analysis and heatmap. Nucleic Acids Res. 2015;43(W1):W566-W570.

44. Friendly M, Fox J. candisc. Version 0.8-5. CRAN. 2021. Accessed February 3, 2021.

45. Kanegaye JT, et al. Recognition of a Kawasaki disease shock syndrome. Pediatrics. 2009;123(5):e783-e789. 